\title{
Radio Emission from Ultrashort-Period Double Degenerate Binaries
}

\author{
A. J. Willes ${ }^{\mathrm{A}, \mathrm{C}}, K . W u^{\mathrm{B}}$, and Z. Kuncic ${ }^{\mathrm{A}}$ \\ A School of Physics, University of Sydney, NSW 2006, Australia \\ ${ }^{B}$ MSSL, University College London, Holmbury St Mary, Surrey, RH5 6NT, UK \\ C E-mail: willes@physics.usyd.edu.au
}

Received 2003 December 8, accepted 2004 January 21

\begin{abstract}
Timing measurements of periodic X-ray pulses from two ultrashort-period double degenerate binaries, RX J1914+24 and RX J0806+15, show that the rates of change of their orbital periods are consistent with gravitational radiation losses. This contradicts the predictions of models which invoke mass transfer between the two white dwarfs. The X-ray emission is, therefore, unlikely to be powered by accretion processes. The unipolar inductor model explains the source of X-ray emission as electrical dissipation at the base of a flux tube, which connects the magnetic white dwarf to its companion. This model is most consistent with the observed X-ray pulse properties. A similar current system exists in the Jupiter-Io system, where a mildly relativistic electron current produces an auroral footprint at the base of the Io flux tube and highly polarized beamed radio emission by means of the electron cyclotron maser mechanism. Detection of radio emission from RX J1914+24 and RX J0806+15 would thus provide further support for the unipolar inductor model. We present theoretical predictions, based on a loss-cone-driven electron cyclotron maser model, of radio fluxes from systems with parameters similar to RX J1914+24 and RX J0806+15.
\end{abstract}

Keywords: masers — radiation mechanisms: non-thermal — stars: binaries: close — stars: white dwarfs — radio continuum: stars

\section{Introduction}

The recent discovery of two ultrashort-period $(P<10 \mathrm{~min})$ X-ray sources, RX J1914+24 (Cropper et al. 1998; Ramsay et al. 2001, 2002b) and RX J0806+15 (Israel et al. 2002; Ramsay, Hakala, \& Cropper 2002a), has confounded existing models of X-ray emitting close binary systems. For systems with orbital periods below $10 \mathrm{~min}$, a Rochelobe filling star will have a density $\sim 4 \times 10^{3} \mathrm{~g} \mathrm{~cm}^{-3}$ (see Frank, King, \& Raine 2002, Chapter 4). These sources are thus proposed to be compact binaries comprising of two degenerate stars, instead of a white dwarf and a mainsequence star (as in Cataclysmic Variables). The peculiar properties of these sources are that both the X-ray and optical emissions possess a single period (interpreted as the orbital period, Cropper et al. 1998) and that these emissions are anti-phased (see Ramsay et al. 2001). Typically, accretion-powered sources display multiple periods at both optical and X-ray wavelengths (corresponding to the orbital rotation, the spin of the accreting star, and their beat period) and exhibit highly excited helium emission lines in their optical spectra. RX J1914+24 and RX J0806+15 lack these characteristic signatures of accreting systems. In addition, accretion-based models generally predict an increase in the orbital period for binary systems with a mass donor star less massive than its compact companion star, when mass loss and angular momentum loss from the systems are insignificant. Precise timing measurements of X-ray pulses from RX J1914+24 (Strohmayer 2002) and optical pulses from RX J0806+15 (Hakala et al.
2003) show instead a gradual decrease in orbital period at a rate consistent with energy losses to gravitational radiation.

Wu et al. (2002) proposed a unipolar inductor model to explain the peculiar X-ray and optical/infrared (IR) variations of RX J1914+24, based on the unipolar inductor model for the Jupiter-Io system (Piddington \& Drake 1968; Goldreich \& Lynden-Bell 1969). In the unipolar inductor model for white dwarf pairs, Jupiter is replaced by a magnetic white dwarf, and Io is replaced by a non-magnetic white dwarf. The X-ray emission is powered by resistive heating of the atmosphere of the magnetic white dwarf, at the footpoints of the magnetic flux tubes which carry the electric currents flowing between the white dwarfs. The X-ray footpoint emission is analogous to the observed ultraviolet auroral footprints of the Galilean moons in Jupiter's atmosphere (Connerney et al. 1993; Clarke et al. 1996, 2002). In the white dwarf unipolar inductor model, the anti-phased optical/IR emission emanates from the irradiated face of the non-magnetic white dwarf, which is visible when the X-ray source is directed away from the observer. The orbital period gradually decreases, as a consequence of orbital angular momentum loss through gravitational radiation. It has also been proposed that unipolar induction can operate in AM Her binaries consisting of a magnetized white dwarf and a red dwarf (Chanmugam \& Dulk 1983) and in isolated magnetic white dwarf systems with terrestrial planets (Li, Ferrario, \& Wickramasinghe 1998). 
Willes \& Wu (2004) proposed that white dwarf unipolar inductor systems, which could be white dwarf pairs or white dwarf planetary systems, should also generate detectable radio emission. The coherent electron cyclotron maser (ECM) emission mechanism produces highly beamed radio emission at the electron gyrofrequency, with high brightness temperatures $\left(T \gg 10^{8} \mathrm{~K}\right)$ and high degrees of circular polarization (typically nearly $100 \%$ ). The ECM mechanism operates in conditions in which the electron cyclotron frequency $\Omega_{\mathrm{e}}$ exceeds the plasma frequency $\omega_{\mathrm{p}}$ (e.g. Dulk 1985) and where there is a source of free energy for wave growth. The convergence of magnetic flux tube field lines near the magnetic white dwarf surface provides the necessary magnetic-field geometry to create a loss cone velocity distribution in the mildly relativistic electrons, which is the assumed source of free energy for wave growth. Willes \& Wu (2004) used a loss-cone-driven ECM emission model to predict radio fluxes from white dwarf unipolar inductor systems.

In this paper we apply the Willes \& Wu model to predict the radio fluxes from white dwarf pairs with parameters similar to RX J1914+24 and RX J0806+15, at frequencies accessible to radio telescopes such as the VLA, ATCA, MERLIN, and Effelsberg. This paper is organized as follows: In Section 2 we briefly outline the maser emission model; in Section 3 we present radio flux predictions for the two candidate white dwarf unipolar inductor systems; and in Section 4 we discuss these results.

\section{Model Outline}

In this section we briefly outline the model used to predict the flux densities for unipolar-inductor-driven radio emission in white dwarf systems, with an emphasis on defining free parameters in the model. A complete model description is provided by Willes \& Wu (2004).

\subsection{The Unipolar Inductor Model}

Figure 1 is a schematic illustration of the white dwarf unipolar inductor system, comprising a magnetic white dwarf with mass, $M_{1}$, and magnetic moment, $\mu$, and a nonmagnetic white dwarf with mass, $M_{2}$. The white dwarf radii, $R_{1}$ and $R_{2}$, are determined from the white dwarf masses using the Hamada \& Salpeter (1961) mass relation. Electron currents are driven in the flux tube current circuit by the asynchronicity between spin and orbital motions in the binary system. The asynchronism parameter, $\xi$, is defined as the ratio of the angular speed of the magnetic white dwarf spin to the orbital angular speed. With nonzero $\xi$, the non-magnetic white dwarf traverses the field lines of the magnetic white dwarf, inducing a potential difference across the highly conducting atmosphere of the non-magnetic white dwarf. The induced currents are carried by mildly relativistic electrons.

The maximum dissipation in the flux tube current circuit occurs in the atmosphere of the magnetic white dwarf at the flux tube footpoints. The cross-section of the flux tube in the atmosphere of the magnetic white dwarf is considerably narrower than that of the non-magnetic

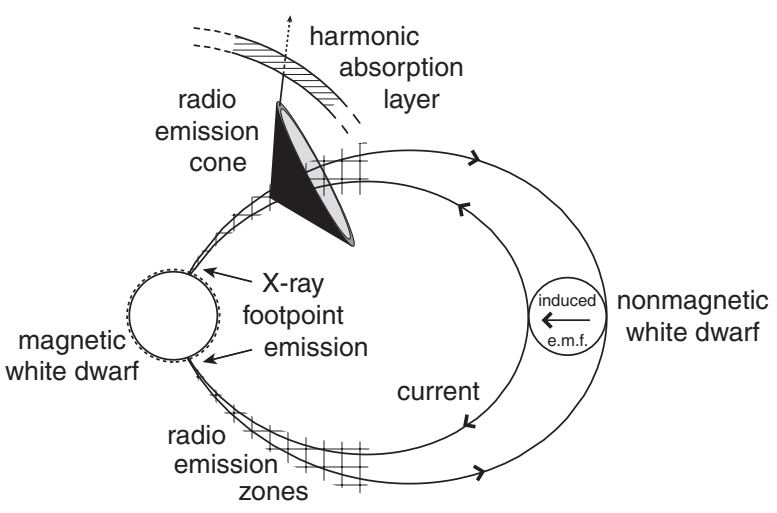

Figure 1 The unipolar inductor model for white dwarf pairs.

white dwarf (see Figure 1). Consequently, the resistivity is substantially higher at the flux tube footpoints in the atmosphere of the magnetic white dwarf. Wu et al. (2002) demonstrated that small values of $\xi$ can produce intense X-ray emissions from the footpoint. For instance, for a white dwarf pair with $M_{1}=1 \mathrm{M}_{\odot}, \mu=10^{32} \mathrm{G} \mathrm{cm}^{3}$, $M_{2}=0.5 \mathrm{M}_{\odot}$, and an orbital period of $10 \mathrm{~min}$, the X-ray luminosity of the footpoint exceeds the solar bolometric luminosity when the degree of asynchronous rotation exceeds $1 \%$.

\subsection{The Maser Emission Model}

The magnetic field lines of the flux tube converge near the magnetic white dwarf surface to provide the ideal geometry for a loss-cone instability to form in the velocity distribution of the mildly relativistic electrons. The loss cone is produced by the preferential magnetic mirroring of large-pitch-angle electrons (Willes \& Wu 2004). The angular width of the loss-cone edge is given the nominal value, $\Delta \alpha=0.05$, in all calculations in this paper. Varying this parameter has only a relatively weak effect on the maser growth rates and flux densities (Willes \& Wu 2004). In this paper we assume that the thermal energy of the electrons in the loss cone is $k_{\mathrm{B}} T_{\mathrm{lc}}=1 \mathrm{keV}$. The electron density in the loss cone, $n_{\mathrm{lc}}$, is defined as the number density of electrons at the mirror point, near the base of the flux tube. The value of $n_{\mathrm{lc}}$ decreases with distance along the flux tube, as the flux tube radius increases, such that the electron density integrated over the cross-section of the flux tube remains constant.

Willes \& Wu (2004) derived an expression for the predicted radio flux densities generated within the unipolar inductor circuit of the white dwarf pair, based on semiquantitative expressions for the loss-cone-driven ECM growth rate, emission bandwidth, and angular ranges, obtained by Melrose \& Dulk (1982). The highest maser growth rates are attained in the fundamental extraordinary and ordinary modes (i.e. at the electron cyclotron frequency). The ECM thus produces $100 \%$ circularly polarized emission. Higher harmonics have considerably lower growth rates and are unlikely to be observed. The peak brightness temperature in the source region is estimated by assuming saturation of the reactive version of 
the ECM instability, i.e. at wave levels where electrons become trapped in the electric field of the growing waves.

Maser radiation encounters harmonic damping as it propagates away from the source region towards decreasing magnetic field strengths. The degree of harmonic damping depends on the thermal (background) electron density, $n_{\text {th }}$, and thermal energy, $k_{\mathrm{B}} T_{\text {th }}$. Harmonic damping effects become negligible for $n_{\text {th }} \lesssim 10^{8} \mathrm{~cm}^{-3}$ and $k_{\mathrm{B}} T_{\mathrm{th}} \lesssim 10 \mathrm{eV}$ (Willes \& Wu 2004).

\section{Results}

Figure 2 displays the maximum flux densities predicted for an observing frequency of $5 \mathrm{GHz}$, assuming model parameters $M_{1}=0.7 \mathrm{M}_{\odot}, M_{2}=0.5 \mathrm{M}_{\odot}, P=300 \mathrm{~s}, n_{\text {th }}=$ $10^{8} \mathrm{~cm}^{-3}, k_{\mathrm{B}} T_{\mathrm{th}}=1 \mathrm{eV}$, and a source-observer distance of $100 \mathrm{pc}$. The main feature is that the maximum flux density scales as $n_{\mathrm{lc}}^{4}$. We assume an upper limit to $n_{\mathrm{lc}}$, such that a maximum of $0.1 \%$ of current electrons contribute to the loss-cone distribution. Flux densities are not displayed beyond this limit. This flux-density cutoff is evident in Figure 2, for $n_{\mathrm{lc}}>10^{8} \mathrm{~cm}^{-3}$. A second flux-density cutoff is evident in Figure 2, at $\mu \simeq 5 \times 10^{29} \mathrm{G} \mathrm{cm}^{3}$. This corresponds to where the observing frequency of $5 \mathrm{GHz}$ exceeds the maximum frequency of maser emission, which is the electron gyrofrequency at the base of the flux tube near the surface of the magnetic white dwarf. For $\mu$ well above this cutoff, the asymptotic dependence of the flux density on the magnetic moment satisfies $\mu^{-7}$. It is clear from Figure 2 that an observing frequency of $5 \mathrm{GHz}$ favours the detection of close white dwarf systems where the magnetic moment approaches, from above, the minimum cutoff value of $\mu \sim 5 \times 10^{29} \mathrm{G} \mathrm{cm}^{3}$. For magnetic moments below this value and these assumed binary parameters, no radio emission from close white dwarf pairs can be detected at $5 \mathrm{GHz}$.

Figure 3 displays the maximum flux densities predicted for an observing frequency of $22 \mathrm{GHz}$. All other parameters retain the same values as in Figure 2. At this observing frequency the minimum cutoff of the magnetic moment shifts upwards to $\mu \sim 2 \times 10^{30} \mathrm{G} \mathrm{cm}^{3}$.

Similar calculations were performed for a lower observing frequency of $500 \mathrm{MHz}$. In this case the peak flux densities over the range of parameter space displayed in Figures 2 and 3 lie below $1 \mathrm{nJy}$.

The peak flux density decreases with increasing orbital period, $P$. Figure 4 shows the peak flux density for $n_{\mathrm{lc}}=10^{7} \mathrm{~cm}^{-3}$ and $\mu=10^{30} \mathrm{G} \mathrm{cm}^{3}$, for four values of $P$ between 5 and $100 \mathrm{~min}$. The peak flux density scales as $P^{-2}$.

\section{Discussion and Conclusions}

The three observing frequencies considered in this paper $(0.5,5$, and $22 \mathrm{GHz})$ are close to the operating frequencies of the MERLIN radio telescope. The Effelsberg telescope can observe at $5 \mathrm{GHz}$, and the VLA at both 5 and $22 \mathrm{GHz}$. In discussing the observability of radio emission from white dwarf pairs it is necessary to consider the source

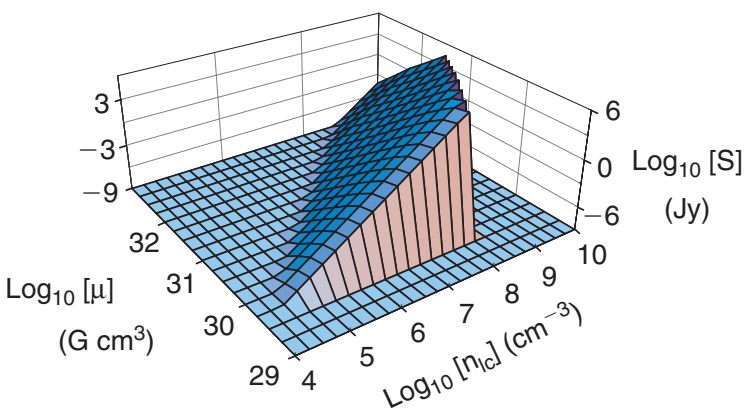

Figure 2 Peak spectral flux densities for maser emission at $5 \mathrm{GHz}$ from a magnetic/non-magnetic white dwarf pair (maximized over emission angle) with system parameters: $P=300 \mathrm{~s}, n_{\text {th }}=10^{8} \mathrm{~cm}^{-3}$ and $k_{\mathrm{B}} T_{\mathrm{th}}=1 \mathrm{eV}$. All plots are calculated for an assumed distance of $100 \mathrm{pc}$.

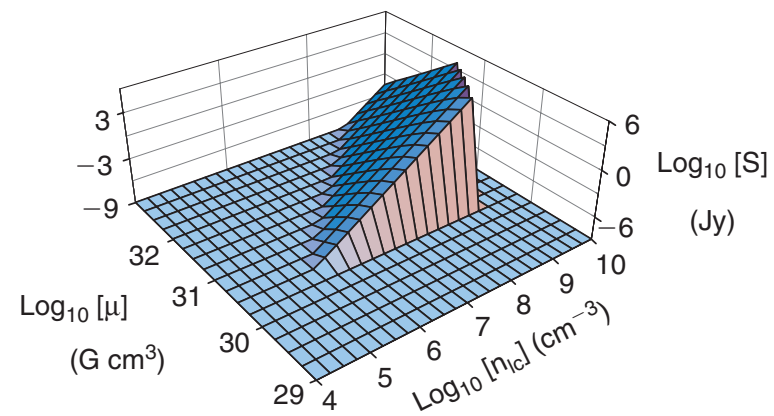

Figure 3 Peak spectral flux densities for maser emission at $22 \mathrm{GHz}$ from a magnetic/non-magnetic white dwarf pair (maximized over emission angle) for the same system parameters as Figure 2.

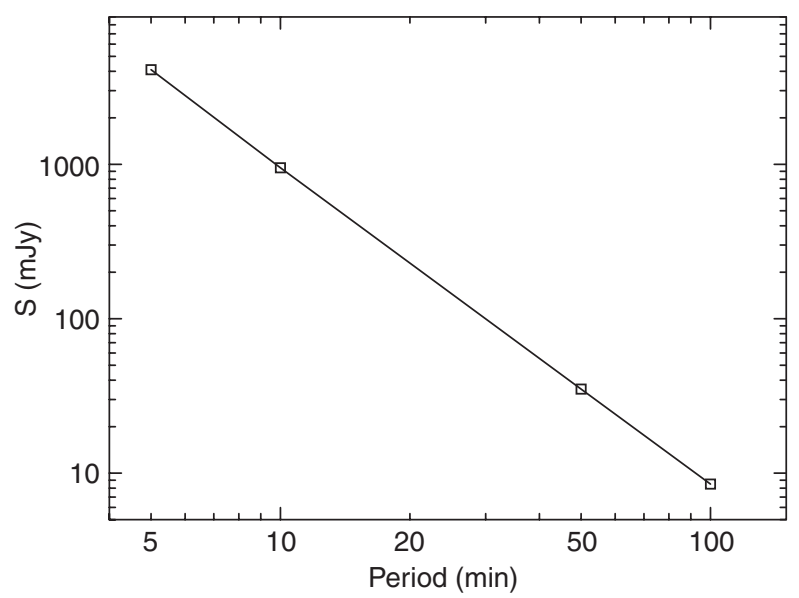

Figure 4 Peak flux density at $5 \mathrm{GHz}$ as a function of $P$ for $n_{\mathrm{lc}}=10^{7} \mathrm{~cm}^{-3}$ and $\mu=10^{30} \mathrm{G} \mathrm{cm}^{3}$.

variability, on orbital period timescales, associated with the beaming of maser radiation. Willes \& Wu (2004) argue that, assuming a pulse duty cycle of a few per cent and observing times on the order of an hour, a detection limit of $1 \mathrm{mJy}$ is attainable with the VLA. Assuming a detection limit of $1 \mathrm{mJy}$, a close white dwarf pair at $100 \mathrm{pc}$ is detectable over a wide range of free parameter space in Figures 2 and 3. These flux-density predictions are readily transformed to other source-observer distances using 
an inverse-square law. Of the three selected observing frequencies $(0.5,5$, and $22 \mathrm{GHz})$, the optimal observing frequency for RX J1914+24 and RX J0806+15 is $5 \mathrm{GHz}$. At this frequency, the flux density increases with decreasing magnetic moment, down to a minimum cutoff at $\mu_{\min } \sim 5 \times 10^{29} \mathrm{G} \mathrm{cm}^{3}$. At the higher observing frequency of $22 \mathrm{GHz}$, the range of observable white dwarf systems is reduced to those systems with magnetic moments exceeding $\sim 2 \times 10^{30} \mathrm{G} \mathrm{cm}^{3}$. We predict no observable radio emission from RX J1914+24 and RX J0806+15 at $500 \mathrm{MHz}$.

Radio emission from ultrashort-period white dwarf binaries will possess a distinctive signature. In addition to pulsed emission from a rotating beamed source, maser emission is generated with high degrees of circular polarisation (nearly 100\%). A further possibility, not explored here, is that the formation of large magnetic-field aligned potential drops (or double layers) close to the surface of the magnetic white dwarf accelerates electrons to relativistic energies, which emit non-thermal synchrotron emission (de Jager 1994; Haerendel 1994; Meintjes \& de Jager 2000). In this case, the beaming is weaker, and the degree of circular polarization is considerably lower. In either case, the radio emission would be easily distinguishable from background galactic sources (with linear polarization). Double layers may also accelerate ions to produce gamma-ray bursts (Meintjes \& de Jager 2000).

\section{Acknowledgments}

The authors thank Gavin Ramsay, for useful discussions, and the referee for bringing the double layer issue to our attention. A.J.W. acknowledges support from the Australian Research Council through an ARC Postdoctoral Research Fellowship.

\section{References}

Chanmugam, G., \& Dulk, G. A. 1983, in Cataclysmic variables and related objects; Proceedings of the Seventy-second Colloquium, Haifa, Israel, August 9-13, 1982 (Dordrecht: Reidel), p. 223

Cropper, M., Harrop-Allin, M. K., Mason, K., Mittaz, J. P., Potter, S. B., \& Ramsay, G. 1998, MNRAS, 293, L57

Clarke, J. T., et al. 1996, Sci, 274, 404

Clarke, J. T., et al. 2002, Natur, 415, 997

Connerney, J. E. P., Baron, R., Satoh, T., \& Owen, T. 1993, Sci, 262, 1035

de Jager, O. C. 1994, ApJS, 90, 775

Dulk, G. A. 1985, ARA\&A, 23, 169

Frank, J., King, A., \& Raine, D. 2002, Accretion Power in Astrophysics (Cambridge: Cambridge University Press)

Goldreich, P., \& Lynden-Bell, D. 1969, ApJ, 156, 59

Haerendel, G. 1994, ApJS, 90, 765

Hamada, T., \& Salpeter, E. 1961, ApJ, 134, 683

Hakala, P., et al. 2003, MNRAS, 343, L10

Israel, G. L., et al. 2002, A\&A, 386, L13

Li, J., Ferrario, L., \& Wickramasinghe, D. T. 1998, ApJ, 503, L151

Meintjes, P. J., \& de Jager, O. C. 2000, MNRAS, 311, 611

Melrose, D. B., \& Dulk, G. A. 1982, ApJ, 259, 844

Piddington, J. H., \& Drake, J. F. 1968, Nature, 217, 935

Ramsay, G., Cropper, M., Wu, K., Mason, K., \& Hakala, P. 2001, MNRAS, 311, 75

Ramsay, G., Hakala, P., \& Cropper, M. 2002, MNRAS, 332, L7

Ramsay, G., Wu, K., Cropper, M., Schmidt, G., Sekiguchi, K., Iwamuro, F., \& Maihara, T. 2002, MNRAS, 333, 575

Strohmayer, T. E. 2002, ApJ, 581, 577

Willes, A. J., \& Wu, K. 2004, MNRAS, 348, 285

Wu, K., Cropper, M., Ramsay, G., \& Sekiguchi, K. 2002, MNRAS, 331,221 